\title{
Reflejo olivococlear contralateral y su relación con ansiedad y calidad de vida en pacientes con tinnitus
}

\section{Contralateral olivocochlear reflex and its relation with anxiety and quality of life in patients with tinnitus}

\author{
Alexis Leiva C. ${ }^{1,5}$, Rodrigo Donoso S. ${ }^{1,5}$, Juan Hernández $I_{.}{ }^{2}$, Paul Délano R. ${ }^{1,3,4,5}$
}

\section{Resumen}

Introducción: La presencia de tinnitus se asocia a un incremento del nivel de ansiedad y empeoramiento de la calidad de vida, sin embargo, aún no existe claridad de si el sistema eferente auditivo podría influir en estas relaciones. Objetivo: Determinar si las amplitudes de las emisiones otoacústicas por producto de distorsión (EOAPD) y el efecto supresor del reflejo olivococlear gatillado con ruido contralateral (ROC) se asocian al grado del impacto psicoemocional y nivel de ansiedad en pacientes con tinnitus. Material y Método: Se evaluaron las amplitudes de EOAPD y magnitud del ROC para cada oído de manera independiente en una cohorte de 32 sujetos tinnitus y 26 controles, cuyos valores fueron correlacionados con State-Trait Anxiety Inventory (STAI) y Tinnitus Handicap Inventory (THI). Resultados: Los pacientes con tinnitus tuvieron una correlación significativa entre la magnitud del ROC del oído izquierdo y los puntajes en las pruebas de STAI y THI. Conclusión: Una mayor carga de ansiedad y peor calidad de vida en sujetos con tinnitus puede estar asociada con un empeoramiento del efecto supresor del ROC de la vía eferente auditiva. Palabras clave: Emisiones otoacústicas, sistema eferente auditivo, tinnitus, reflejo olivococlear, ansiedad, calidad de vida.

\begin{abstract}
Introduction: Tinnitus is associated with an increase in the degree of anxiety and with worse quality of life. However, whether there are relations between the function of the auditory efferent system and anxiety and tinnitus distress levels is not known. Aim: To determine possible associations between the distortion product otoacoustic emission (DPOAE) amplitudes and the suppressing effect of the olivocochlear reflex (OCR) with anxiety and psycho-emotional impact in tinnitus. Material and Method: DPOAE amplitudes and OCR strength were evaluated for each ear independently in a cohort of 32 tinnitus and 26 controls, whose values were correlated with the State-Trait Anxiety Inventory (STAI) and Tinnitus Handicap Inventory (THI). Results: A significant correlation of STAI and THI scales with the OCR strength measured in the left ear was found in subjects with tinnitus. Conclusion: A higher anxiety load and worse quality of life in subjects with tinnitus may be associated with an impaired olivocochlear reflex suppressing effect. Keywords: otoacoustic emissions, auditory efferent, tinnitus, olivocochlear reflex, anxiety, quality of life.
\end{abstract}

'Departamento de Neurociencias, Facultad de Medicina, Universidad de Chile. Santiago, Chile. ${ }^{2}$ Centro de Estudios Audiológicos, DETEC. Santiago, Chile. ${ }^{3}$ Departamento de Otorrinolaringología, Hospital Clínico de la Universidad de Chile. Santiago, Chile. ${ }^{4}$ Centro Avanzado de Ingeniería Eléctrica y Electrónica, AC3E, Universidad Técnica Federico Santa María. Valparaíso, Chile.

${ }^{5}$ Biomedical Neuroscience Institute, BNI, Facultad de Medicina, Universidad de Chile. Santiago, Chile.

Los autores declaran no tener conflictos de interés. Este trabajo fue financiado por Proyecto Fondecyt 1161155, ANID Fondo Basal FB008, Proyecto ICN09_015, Financiado por la Vicerrectoría de Investigación y Desarrollo (VID) de la Universidad de Chile, código proyecto: ENL 19/20, y Fundación Guillermo Puelma.

Recibido el 19 de agosto de 2020. Aceptado el 30 de agosto de 2020.

Correspondencia:

Paul Délano R.

Departamento Otorrinolaringología, Hospital Clínico Universidad de Chile Santos Dumont 999. Santiago, Chile. Email:pdelano@med.uchile.cl

\section{Introducción}

El tinnitus es la percepción de sonidos en ausencia de una fuente sonora externa ${ }^{1}$. Su prevalencia en la población general, según diversos estudios epidemiológicos, varía desde $5,1 \%$ a $42,7 \%{ }^{2}$. Dentro de este grupo existe una cantidad no menor de pacientes en el cual el tinnitus se ha relacionado con una disminución significativa en su calidad de vida, aso- 
ciado a una mayor prevalencia de depresión, irritabilidad, ansiedad, problemas atencionales y mayores tasas de suicidio ${ }^{3,4}$, y cuya forma de presentación clínica más frecuente es el tinnitus crónico no pulsátil (TCNP), el cual puede ser de presentación uni o bilateral ${ }^{1,5}$.

El origen del TCNP se centraría en la pérdida auditiva periférica (o desaferentación) lo cual genera una hiperactividad compensatoria en diversas estructuras de la vía auditiva central, aumentando la ganancia de sus respuestas, especialmente a nivel de los colículos inferiores y corteza auditiva ${ }^{6}$. Además, para que el tinnitus sea percibido como un síntoma molesto deben ocurrir una serie de alteraciones tanto a niveles corticales y subcorticales del cerebro que afectan a redes neuronales involucradas en funciones cognitivas y el procesamiento emocional ${ }^{7,8}$.

El sistema eferente auditivo es una red neuronal que puede influenciar la mecánica coclear a través de vías descendentes que hacen sinapsis con el complejo olivar superior $(\mathrm{COS})^{9,10}$. En el COS se ubican las neuronas olivococleares mediales (COSM) que inervan a las células ciliadas externas (CCE) de la cóclea $^{11,12}$. La activación del sistema olivococlear suprime al amplificador coclear de las $C C E^{13,14}$. Los estudios que han intentado relacionar la función del sistema eferente auditivo con la presencia de tinnitus no son concluyentes, ya que existen trabajos que avalan una disfunción eferente en tinnitus, pero otros no encuentran ninguna relación ${ }^{15,16}$.

La asociación entre tinnitus y ansiedad estaría mediada por estructuras y circuitos cerebrales que interactúan con la vía auditi$\mathrm{va}^{17}$. Uno de los núcleos más importantes del cerebro en la fisiopatología de la ansiedad es el locus coeruleus (LC). El LC es un núcleo noradrenérgico del tronco encefálico que está implicado en el circuito del nivel de alerta y su disfunción se asocia a trastornos ansiosos ${ }^{18-20}$. Desde el punto de vista neuroanatómico, el LC se conecta con diversas estructuras de la vía auditiva central, incluyendo neuronas fusiformes de los núcleos cocleares dorsales ${ }^{21}$, neuronas olivococleares del sistema eferente auditivo ${ }^{22,23}$ y neuronas de la corteza auditiva ${ }^{24,25}$. De esta forma la hiperactividad del LC podría estar involucrada en el aumento de ansiedad en pacientes con tinnitus.
Estudios de inmunohistoquímica e inmunofluorescencia en roedores han evidenciado que el COS recibe inervación noradrenérgica directa del $\mathrm{LC}^{22,23}$, junto a la evidencia de que la inyección de noradrenalina en las vías auditivas del tronco encefálico generaría un efecto inhibitorio sobre el output coclear al estimular el $\operatorname{COSM}^{23}$. A partir de este marco teórico surge la pregunta de investigación: ¿estará el COSM involucrado en la presencia de ansiedad en pacientes con tinnitus? ¿existirá una relación entre la magnitud del reflejo olivococlear y los niveles de ansiedad o trastornos psicoemocionales de pacientes con tinnitus? En este trabajo proponemos como hipótesis que la función del reflejo olivococlear medial -medida con ruido de banda ancha contralateral y emisiones otoacústicas por producto de distorsión (EOAPD)se correlaciona con el nivel de ansiedad y de calidad de vida en pacientes con tinnitus y umbrales auditivos normales.

\section{Objetivo}

Determinar si la ansiedad y la calidad de vida en pacientes con tinnitus se correlacionan con la variabilidad interindividual del reflejo olivococlear medial medido a través de la supresión de amplitud de las emisiones otoacústicas por productos de distorsión en presencia de ruido de banda ancha contralateral.

\section{Material y Método}

Estudio prospectivo, de corte transversal, de casos (tinnitus) y controles. Ambos grupos de estudio correspondieron a pacientes del Hospital Clínico de la Universidad de Chile y del Centro de Estudios Audiológicos DETEC reclutados entre los años 2017 y 2019. Los pacientes fueron invitados a participar voluntariamente en el estudio y todos firmaron un consentimiento informado. Todos los procedimientos realizados en este trabajo fueron aprobados por el Comité de Ética de Investigación del Hospital Clínico de la Universidad de Chile (número de aprobación OAIC 016/20042016). Los criterios de inclusión dentro del grupo de casos con tinnitus fueron: edad entre 18 y 60 años, tinnitus crónico no pulsátil ( $>3$ meses) unilateral o bilateral y audiometría con promedio tonal puro (PTP en 0,5, 1, 2 y $4 \mathrm{kHz}$ ) 
$\leq 25 \mathrm{~dB}$. Se realizó una impedanciometría para descartar compromiso del oído medio y estudio audiométrico de $125 \mathrm{~Hz}$ a $16 \mathrm{kHz}$.

\section{Tinnitus hándicap inventory (THI)}

Para evaluar el impacto psicológico, social e incapacidad del tinnitus en el paciente se aplicó el tinnitus hándicap inventory (THI ${ }^{26-28}$, el cual consiste en 25 preguntas con tres opciones de respuesta cada una: (i) sí, (ii) a veces y (iii) no, otorgándole 4 puntos al sí, 2 puntos al a veces, y 0 puntos al no, de manera que la suma del puntaje total final puede ir de 0 a 100 . Este puntaje se utilizó para dividir en 5 niveles el grado de afectación por el tinnitus: grado 1, muy leve (THI 0 a 16); grado 2, leve (THI 18 a 36); grado 3 , moderado (THI 38 a 56); grado 4 , severo (THI 58 a 76) y grado 5 , catastrófico (THI 78 a 100$)^{29}$.

\section{State-trait anxiety inventory (STAI)}

Se utilizó el cuestionario de ansiedad estado-rasgo ${ }^{30,31}$ (state-trait anxiety inventory). Este instrumento tiene dos escalas de autoevaluación para medir dos conceptos independientes de la ansiedad: estado y rasgo, sin embargo, para nuestro estudio nos enfocamos en evaluar la ansiedad-rasgo, la cual está definida como una propensión ansiosa estable, propia del sujeto, que hace percibir a las personas y a las situaciones como amenazadoras. Ambas escalas de ansiedad contienen un total de 20 ítems de preguntas, graduadas de 1 a 4 según su severidad, correspondiendo un puntaje mayor un nivel más alto de ansiedad. En la escala ansiedad-rasgo las respuestas son: 1) casi nunca; 2) algunas veces; 3 ) frecuentemente y 4) casi siempre.

\section{Medición de emisiones otoacústicas por producto de distorsión y reflejo olivococlear con ruido contralateral}

Se midieron las EOAPD (2f1-f2) utilizando un micrófono ER10C (Etymotic Research), presentando ocho pares de tonos primarios (f1 y f2, a 65 y $55 \mathrm{~dB}$ SPL, relación f2/f1 de $1,22)$ en cada oído. Obtuvimos ocho frecuencias diferentes de EOAPD (2f1-f2): 707, 891, $1.122,1.414,1.781,2.244,2.828$ y $3.563 \mathrm{~Hz}$. El protocolo incluyó tres bloques: en los primeros dos bloques se midieron solo las amplitudes de las EOAPD en el oído ipsilateral sin presencia de ruido contralateral. En el tercer bloque se midieron las amplitudes de las EOAPD en presencia de un ruido de banda ancha contralateral a $60 \mathrm{~dB}$ SPL. El tiempo de presentación de cada par de tonos ( $\mathrm{f} 1 \mathrm{y} \mathrm{f2}$ ) fue de un segundo. El tiempo entre estímulos fue de 0,5 segundos. Este proceso se repitió seis veces, por lo que contamos con 144 valores que se promediaron en cada caso. Para considerar una EOAPD como presente se utilizó un criterio de amplitud en que la EOAPD tenía que ser al menos $6 \mathrm{~dB}$ SPL sobre el ruido de fondo. En este protocolo las amplitudes de las EOAPD con y sin ruido contralateral fueron registradas y analizadas para ambos oídos de manera independiente, por otro lado, el efecto supresor de la fuerza del reflejo olivocococlear (ROC) se calculó como la diferencia entre las amplitudes de las EOAPD por cada frecuencia obtenidas en las condiciones sin ruido contralateral menos con ruido contralateral en dB SPL, de manera que los resultados serán visualizados con valores negativos. Así, un mayor efecto supresor del ROC será equivalente a un valor negativo de mayor magnitud en el análisis y gráficos. Se compararon las amplitudes de EOAPD y la magnitud del ROC entre pacientes con tinnitus y sujetos controles.

\section{Análisis estadístico}

Se realizó mediante programa SPSS Statistics 20, en la cual se ocuparon medidas de estadística descriptiva y pruebas paramétricas para hacer comparaciones de variables entre los grupos de estudio por oído mediante t-student, y no paramétricas mediante test de Mann-Whitney o Wilcoxon, mientras que las comparaciones múltiples fueron analizadas mediante un test de ANOVA de un factor. Las correlaciones de variables audiológicas con test psicométricos fueron mediante el test de Pearson para medidas paramétricas y test de Spearman para no paramétricas. Para cada caso y para cada test se consideró un $p$ value $<0,05$ para determinar significancia estadística.

\section{Resultados}

Se reclutaron 32 pacientes con tinnitus y umbrales audiométricos $\leq 25 \mathrm{~dB}$ (500$4.000 \mathrm{~Hz}$ ) (9 mujeres, $28,1 \%$ ) y 26 sujetos controles (11 mujeres, $34,4 \%$ ). La edad pro- 
medio del grupo con tinnitus fue de 40,3 \pm 11,8 años (promedio \pm desviación estándar) $y$ en el grupo de controles fue de 34,2 $\pm 9,1$ años, diferencia estadísticamente significativa ( $t$-student, $\mathrm{p}=0,03)$. Respecto a la lateralidad de percepción del tinnitus, fue bilateral en 12 sujetos (37,5\%), solo en oído izquierdo para 11 sujetos $(34,4 \%)$, y solo en oído derecho para 9 sujetos $(28,1 \%)$.

En la Figura 1 se pueden observar los umbrales audiométricos promedios entre 125 y 16

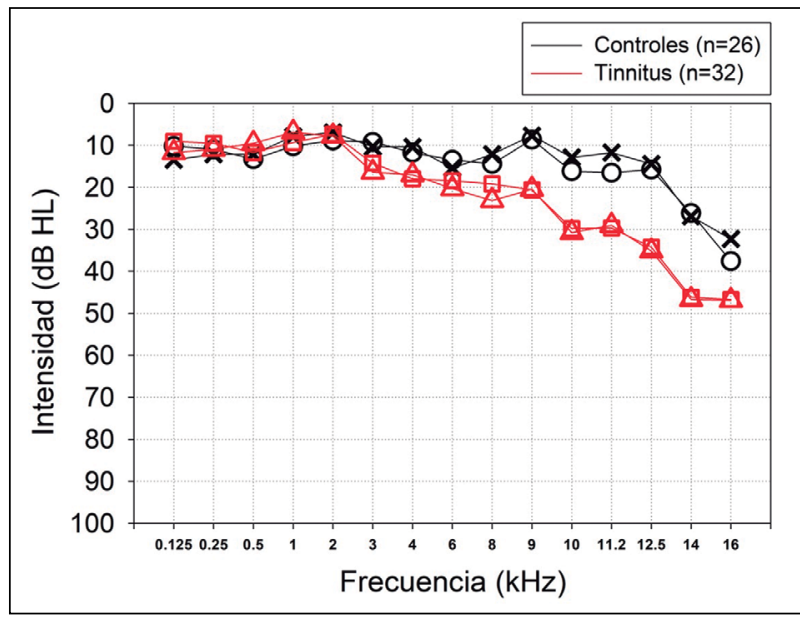

Figura 1. Audiometría de alta frecuencia promedio de tinnitus (rojo) y controles (negro). Los oídos derechos se muestran con triángulos y círculos, y los oídos izquierdos con cruces y cuadrados. Los pacientes con tinnitus tienen peores umbrales que los controles para frecuencias de 9 a $16 \mathrm{kHz}$.
kHz. Los PTP $(0,5-4 \mathrm{kHz})$ de la audiometría en el grupo de tinnitus fueron: $9,5 \pm 5,7 \mathrm{~dB}$ en oído derecho y $8,1 \pm 5,6$ dB en el oído izquierdo, mientras que para el grupo control fueron de: 10,8 $\pm 5,5 \mathrm{~dB}$ en el oído derecho y 9,0 \pm 5,2 $\mathrm{dB}$ en el oído izquierdo. No hubo diferencias significativas entre el PTP $(0,5-4 \mathrm{kHz})$ del grupo control y tinnitus ( $t$-student, oído derecho: $\mathrm{p}=0,62$; oído izquierdo: $\mathrm{p}=0,50)$. Por otro lado, los umbrales de alta frecuencia (9-16 $\mathrm{kHz}$ ) fueron significativamente más elevados en el grupo con tinnitus (tinnitus: $34,8 \pm 24,4$ $\mathrm{dB}$ en el oído derecho y $35,1 \pm 23,7 \mathrm{~dB}$ en el oído izquierdo; controles: $20,4 \pm 17,3 \mathrm{~dB}$ en el oído derecho y 17,8 dB $\pm 13,6 \mathrm{~dB}$ en el oído izquierdo; $t$-student, oído derecho: $\mathrm{p}=0,01$; oído izquierdo: $\mathrm{p}=0,007$ ).

En la Tabla 1 se muestran los valores promedios de las amplitudes de EOAPD para cada una de las ocho frecuencias utilizadas (f2). El grupo con tinnitus presentó una reducción significativa de la amplitud de las EOAPD para las frecuencias de 2.786 $\mathrm{Hz}$ (oído derecho: $\mathrm{p}=0,04$; oído izquierdo: $\mathrm{p}=0,007)$ y $3508 \mathrm{~Hz}$ (oído derecho: $\mathrm{p}=0,03$; oído izquierdo: $\mathrm{p}=0,04$ ) (Figura 2). Por otro lado, en la Tabla 2 se puede visualizar que no hubo diferencias significativas entre el grupo tinnitus y control al evaluar el efecto del reflejo olivococlear evocado con ruido contralateral sobre la amplitud de las EOAPD para ninguna de las frecuencias estudiadas.

Tabla 1. Amplitudes de EOAPD (en dB SPL) en grupo de tinnitus y controles

\begin{tabular}{|c|c|c|c|c|c|c|}
\hline \multirow[b]{2}{*}{ f2 } & \multicolumn{2}{|c|}{ Tinnitus } & \multicolumn{2}{|c|}{ Controles } & \multirow[b]{2}{*}{$\begin{array}{c}\text { p value } \\
\text { OD }\end{array}$} & \multirow[b]{2}{*}{$\begin{array}{c}\text { p value } \\
\text { Ol }\end{array}$} \\
\hline & $\begin{array}{c}O D \\
\text { Prom } \pm D E\end{array}$ & $\begin{array}{c}\text { OI } \\
\text { Prom } \pm \text { DE }\end{array}$ & $\begin{array}{c}O D \\
\text { Prom } \pm \text { DE }\end{array}$ & $\begin{array}{c}\text { OI } \\
\text { Prom } \pm \text { DE }\end{array}$ & & \\
\hline $1105 \mathrm{~Hz}$ & $8,81 \pm 6,88$ & $9,35 \pm 5,77$ & $7,11 \pm 7,20$ & $10,69 \pm 6,78$ & 0,20 & 0,45 \\
\hline $1393 \mathrm{~Hz}$ & $9,27 \pm 7,18$ & $10,01 \pm 6,13$ & $9,90 \pm 5,98$ & $9,59 \pm 6,63$ & 0,73 & 0,81 \\
\hline $1754 \mathrm{~Hz}$ & $6,61 \pm 7,26$ & $8,44 \pm 6,59$ & $7,55 \pm 4,94$ & $8,56 \pm 4,94$ & 0,56 & 0,93 \\
\hline $2210 \mathrm{~Hz}$ & $4,17 \pm 7,02$ & $3,92 \pm 7,69$ & $5,57 \pm 5,72$ & $6,40 \pm 4,90$ & 0,42 & 0,16 \\
\hline $2786 \mathrm{~Hz}$ & $0,61 \pm 6,22$ & $0,77 \pm 6,44$ & $4,04 \pm 5,86$ & $5,33 \pm 5,11$ & $0,04^{*}$ & $0,007 * *$ \\
\hline $3508 \mathrm{~Hz}$ & $1,16 \pm 6,48$ & $1,97 \pm 6,93$ & $4,16 \pm 6,56$ & $5,43 \pm 5,78$ & $0,03 *$ & $0,04^{*}$ \\
\hline $3624 \mathrm{~Hz}$ & $2,05 \pm 7,04$ & $2,24 \pm 7,20$ & $3,73 \pm 6,33$ & $5,56 \pm 6,75$ & 0,38 & 0,09 \\
\hline $4568 \mathrm{~Hz}$ & $3,13 \pm 6,10$ & $1,90 \pm 7,66$ & $2,19 \pm 7,24$ & $3,70 \pm 6,33$ & 0,64 & 0,38 \\
\hline
\end{tabular}



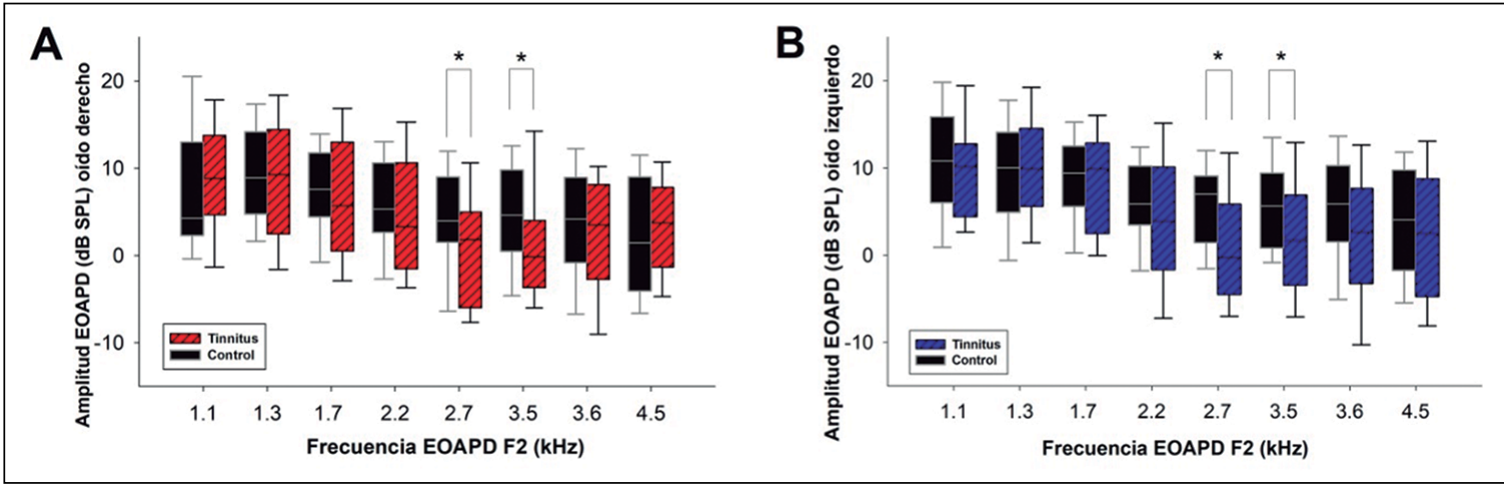

Figura 2. Amplitud de emisiones otoacústicas por producto de distorsión en tinnitus y controles. A. EOAPD del oído derecho. B. EOAPD del oído izquierdo. Se observan diferencias significativas en las frecuencias de 2.786 y $2.508 \mathrm{~Hz}$ en forma bilateral $\left({ }^{*} p<0,05\right)$.

\begin{tabular}{|c|c|c|c|c|c|c|}
\hline \multirow[b]{2}{*}{ f2 } & \multicolumn{2}{|c|}{ Tinnitus } & \multicolumn{2}{|c|}{ Controles } & \multirow[b]{2}{*}{$\begin{array}{c}p \text { value } \\
\text { OD }\end{array}$} & \multirow[b]{2}{*}{$\begin{array}{c}\text { p value } \\
\text { OI }\end{array}$} \\
\hline & $\begin{array}{c}\text { OD } \\
\text { Prom } \pm \text { DE }\end{array}$ & $\begin{array}{c}\text { Ol } \\
\text { Prom } \pm \text { DE }\end{array}$ & $\begin{array}{c}\text { OD } \\
\text { Prom } \pm \text { DE }\end{array}$ & $\begin{array}{c}\text { OI } \\
\text { Prom } \pm \text { DE }\end{array}$ & & \\
\hline $1105 \mathrm{~Hz}$ & $-0,75(0,89)$ & $-0,76(1,28)$ & $-0,63(1,23)$ & $-0,62(1,00)$ & 0,78 & 0,68 \\
\hline $1393 \mathrm{~Hz}$ & $-0,56(0,69)$ & $-0,92(0,76)$ & $-0,73(1,07)$ & $-0,59(1,16)$ & 0,77 & 0,36 \\
\hline $1754 \mathrm{~Hz}$ & $-0,76(1,10)$ & $-1,03(1,07)$ & $-0,57(0,83)$ & $-0,50(0,95)$ & 0,49 & 0,06 \\
\hline $2210 \mathrm{~Hz}$ & $-0,45(0,86)$ & $-0,41(1,20)$ & $-0,70(1,20)$ & $-0,62(0,80)$ & 0,36 & 0,46 \\
\hline $2786 \mathrm{~Hz}$ & $-0,43(1,12)$ & $-0,46(1,21)$ & $-0,36(0,88)$ & $-0,47(0,61)$ & 0,80 & 0,95 \\
\hline $3508 \mathrm{~Hz}$ & $-0,69(1,19)$ & $-0,23(0,87)$ & $-0,33(0,86)$ & $-0,31(0,46)$ & 0,22 & 0,68 \\
\hline $3624 \mathrm{~Hz}$ & $-0,12(0,68)$ & $-0,32(0,58)$ & $-0,41(0,62)$ & $-0,19(0,74)$ & 0,12 & 0,98 \\
\hline $4568 \mathrm{~Hz}$ & $-0,19(0,56)$ & $-0,01(0,87)$ & $-0,14(0,90)$ & $-0,27(0,56)$ & 0,26 & 0,58 \\
\hline
\end{tabular}

No hubo diferencias estadísticamente significativas. $\mathrm{OD}=$ oído derecho, $\mathrm{Ol}=$ oído izquierdo, Prom = promedio, $\mathrm{DE}=$ desviación estándar.

El puntaje promedio del THI en los pacientes con tinnitus fue de 31,25 $\pm 19,22$ puntos (mediana 26) y al agruparlos según la escala de severidad del THI tuvimos un total de 20 sujetos en el grupo "leve-muy leve" y 12 sujetos en el grupo "moderado-severo". En nuestro estudio ningún sujeto obtuvo el puntaje requerido para ser catalogado como parte del grupo catastrófico. En relación a la medición de ansiedad, el puntaje de la encuesta STAI fue de 37,56 \pm 9,28 (mediana: 35 ) en pacientes con tinnitus y de 35,92 $\pm 11,31$ (mediana: 32 ) en sujetos controles (diferencia no significativa, Mann-Whitney, $\mathrm{p}=0,33$ ). En la agrupación de sujetos con tinnitus cuyos puntajes de escala de severidad del THI eran "leve-muy leve" y "moderado-severo" sus puntajes del STAI fueron de 33,25 $\pm 6,99$ (mediana: 31 ) y 44,75 $\pm 8,26$ (mediana: 45) (diferencia significativa, Mann-Whitney, $\mathrm{p}=0,02)$. Las amplitudes de las EOAPD en el grupo THI "leve-muy leve" fueron de 4,89 $\pm 4,79 \mathrm{~dB}$ SPL en el oído derecho y de 4,18 $\pm 5,75 \mathrm{~dB}$ SPL en el oído izquierdo, mientras que en el grupo THI "moderado-severo" fueron de 3,43 $\pm 5,64$ dB SPL en el oído derecho y de 5,09 $\pm 4,53 \mathrm{~dB}$ SPL en el oído izquierdo. Estas diferencias no fueron significativas (oído derecho: $t$-student, $\mathrm{p}=0,44$; oído izquierdo: $t$-student, $\mathrm{p}=0,64$ ).

Por otro lado, para el efecto supresor del ROC, el grupo THI "leve-muy leve" tuvo un promedio de $-0,59 \pm 0,45 \mathrm{~dB}$ SPL en el oído 
derecho y -0,81 $\pm 0,34 \mathrm{~dB}$ SPL en el oído izquierdo, mientras que en el grupo "moderado-severo" fueron de $-0,41 \pm 0,36 \mathrm{~dB}$ SPL en el oído derecho y de $-0,10 \pm 0,35 \mathrm{~dB}$ SPL en el oído izquierdo. El efecto supresor del ROC en el oído izquierdo fue significativamente menor en el grupo THI "moderado-severo" en relación al grupo "leve-muy leve" ( $t$-student, $\mathrm{p}<0,01)$. No hubo diferencias del ROC entre los mismos grupos THI en el oído derecho ( $t$-student, $\mathrm{p}=0,25)$.

Se puede observar en la Tabla 3 que al realizar la comparación múltiple de los promedios del efecto supresor del ROC medido en el oído izquierdo entre el grupo control y los grupos de tinnitus agrupados según THI en "leve-muy leve" y "moderado-severo" se encontró una diferencia significativa entre los tres grupos (ANOVA, F $(2,54)=17,22$ y $\mathrm{p}<0,01)$. La comparación post-hoc usando el test de Bonferroni reveló que la amplitud de las EOAPD del grupo control era significativamente mayor sobre el grupo de tinnitus "moderado-severo" ( $p=0,013)$ y significativamente menor comparado al grupo "leve-muy leve” $(\mathrm{p}=0,02)$. Las comparaciones de ambos grupos de sujetos con tinnitus y el efecto supresor del ROC medido en el oído derecho no evidenciaron diferencias significativas $(F$ $(2,55)=0,77$ y $\mathrm{p}=0,47)$.

Luego correlacionamos las amplitudes de las EOAPD y el efecto del ROC con los puntajes en las encuestas de THI y STAI. La Tabla 4 muestra un resumen de estos resultados, evidenciando una correlación entre la magnitud del ROC del oído izquierdo y el puntaje STAI y THI en los pacientes con tinnitus. No hubo correlaciones significativas para el oído derecho. En la Figura 3 se muestran los valores individuales de las correlaciones de la magnitud del ROC del oído izquierdo y derecho con los puntajes de las pruebas THI y STAI.

Tabla 3. Comparación de las amplitudes de las EOAPD y efecto supresor del ROC promedios entre los grupos control y de tinnitus subdividido de acuerdo a escala de severidad del THI

\begin{tabular}{|c|c|c|c|c|}
\hline $\begin{array}{l}\text { Variables de } \\
\text { análisis }\end{array}$ & $\begin{array}{c}\text { Control } \\
\text { Prom } \pm \text { DE }\end{array}$ & $\begin{array}{l}\text { Tinnitus L-ML } \\
\text { Prom } \pm \text { DE }\end{array}$ & $\begin{array}{l}\text { Tinnitus M-S } \\
\text { Prom } \pm \text { DE }\end{array}$ & $\begin{array}{l}\text { Test de ANOVA: diferencias } \\
\text { entre los tres grupos }\end{array}$ \\
\hline AMP OD & $5,19 \pm 4,85$ & $4,89 \pm 4,79$ & $3,43 \pm 5,64$ & $F(2,55)=0,53 ; p=0,59$ \\
\hline AMP OI & $6,60 \pm 4,65$ & $4,18 \pm 5,75$ & $5,09 \pm 4,53$ & $F(2,54)=1,31 ; p=0,28$ \\
\hline $\mathrm{ROC} O \mathrm{OD}$ & $-0,44 \pm 0,52$ & $-0,59 \pm 0,45$ & $-0,41 \pm 0,36$ & $F(2,55)=0,77 ; p=0,47$ \\
\hline ROC Ol & $-0,45 \pm 0,32$ & $-0,81 \pm 0,34$ & $-0,10 \pm 0,35$ & $F(2,54)=17,22 ; p<0,01 * *$ \\
\hline
\end{tabular}

Sólo se encontró una diferencia significativa en el ROC del grupo severo-moderado del oído izquierdo tanto para el test de ANOVA como la evaluación post-hoc de Bonferroni $\left({ }^{*} p<0,01\right)$. AMP $=$ amplitud, ROC $=$ reflejo olivococlear, OD = oído derecho, OI = oído izquierdo. L-ML: Escala de severidad THI leve-muy leve. M-S: Escala de severidad THI moderado-severo.

Tabla 4. Correlaciones de Spearman entre EOAPD basal, reflejo olivococlear en EOAPD, STAI y THI

\begin{tabular}{|c|c|c|c|c|c|c|c|}
\hline & \multicolumn{7}{|c|}{ Escala de severidad del THI } \\
\hline & \multicolumn{3}{|c|}{ Independiente } & \multicolumn{2}{|c|}{ Leve-Muy leve } & \multicolumn{2}{|c|}{ Moderado-Severo } \\
\hline & $\begin{array}{c}\text { THI } \\
\text { Tinnitus }\end{array}$ & $\begin{array}{c}\text { STAI } \\
\text { Tinnitus }\end{array}$ & $\begin{array}{c}\text { STAI } \\
\text { controles }\end{array}$ & $\begin{array}{c}\text { THI } \\
\text { Tinnitus }\end{array}$ & $\begin{array}{c}\text { STAI } \\
\text { Tinnitus }\end{array}$ & $\begin{array}{c}\text { THI } \\
\text { Tinnitus }\end{array}$ & $\begin{array}{c}\text { STAI } \\
\text { Tinnitus }\end{array}$ \\
\hline AMP OD & $-0,11$ & $-0,03$ & $-0,19$ & $-0,13$ & $-0,10$ & 0,25 & 0,28 \\
\hline AMP OI & 0,18 & 0,12 & 0,04 & $-0,01$ & 0,01 & 0,45 & 0,25 \\
\hline ROC OD & 0,07 & $-0,01$ & 0,02 & $-0,14$ & 0,03 & $-0,24$ & $-0,06$ \\
\hline $\mathrm{ROC} \mathrm{OI}$ & $0,66^{*}$ & $0,61 *$ & $-0,16$ & $-0,18$ & 0,30 & 0,53 & 0,33 \\
\hline
\end{tabular}

Los pacientes con tinnitus fueron, además, agrupados según la severidad del THI. Se muestran los coeficientes de correlación ( $r$ ) correspondientes. El ROC OI fue significativo con los puntajes de THI y STAI $\left({ }^{*} p<0,05\right)$. AMP $=$ amplitud, ROC $=$ reflejo olivococlear, $\mathrm{OD}$ = oído derecho, OI = oído izquierdo. 

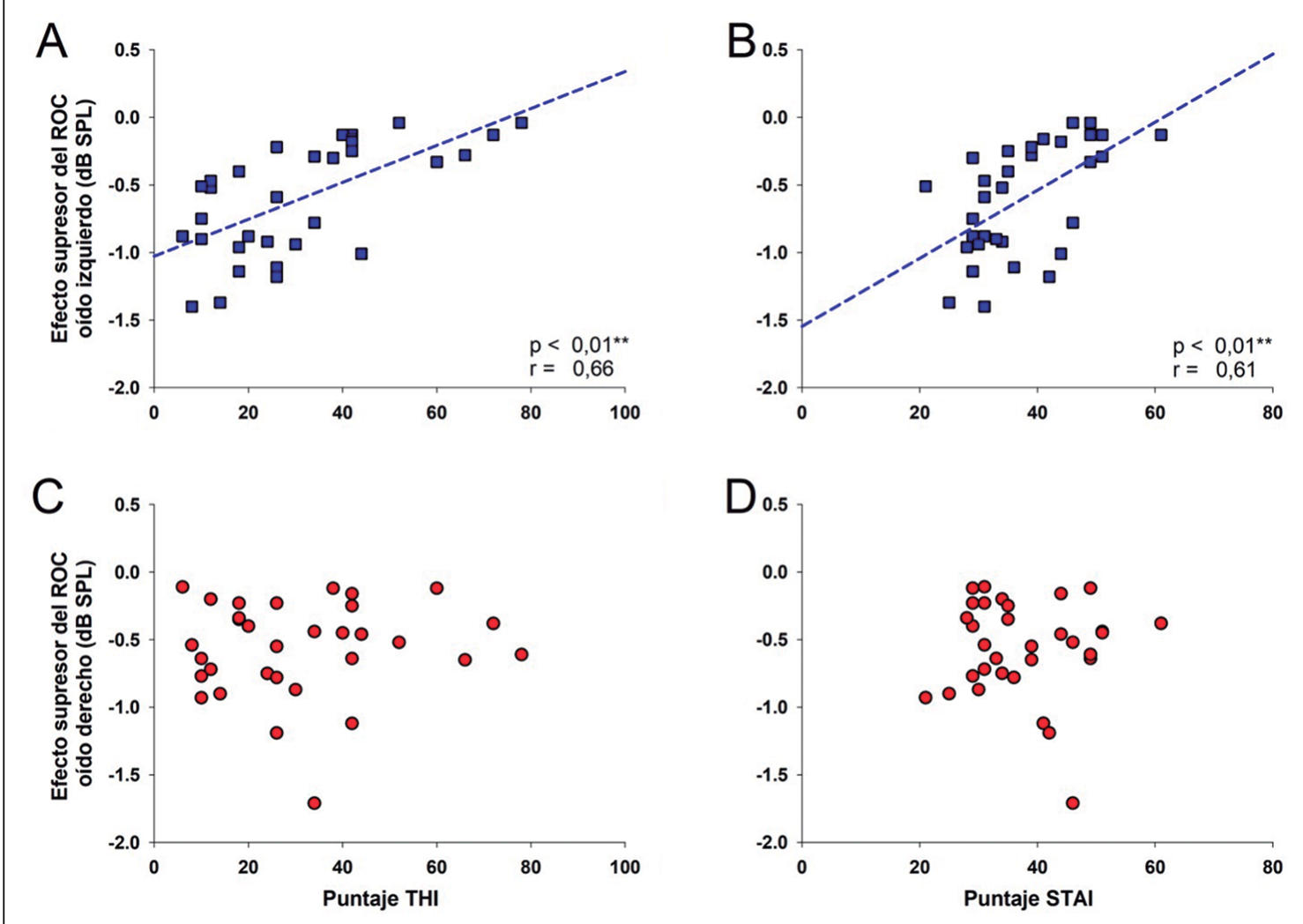

Figura 3. Relación entre magnitud del reflejo olivococlear contralateral y puntajes THI y STAI en pacientes con tinnitus. El oído izquierdo y derecho se grafican en azul y rojo respectivamente. La magnitud del ROC del Ol se correlaciona con A. El puntaje THI y B. El puntaje STAI. C. y D. No hubo correlación del ROC del OD con C. El puntaje THI ni con D. El puntaje STAI.

\section{Discusión}

A modo de resumen, en este estudio encontramos que los pacientes con tinnitus y PTP normal $(0,5$ a $4 \mathrm{kHz})$ presentaron una disminución de los umbrales auditivos de alta frecuencia ( 9 a $16 \mathrm{kHz}$ ), de las amplitudes de las EOAPD en frecuencias de 2,7 y 3,5 kHz, y correlaciones significativas entre la magnitud del ROC del oído izquierdo con los puntajes de las pruebas STAI y THI.

\section{Umbrales audiométricos de alta frecuencia en tinnitus y PTP normal}

A pesar de que los pacientes con tinnitus fueron reclutados con el criterio de tener PTP normal de 0,5 a $4 \mathrm{kHz}$, se puede observar que en los umbrales de alta frecuencia $(9 \mathrm{a} 16 \mathrm{kHz})$ se produce una reducción bilateral mayor en los sujetos con tinnitus comparados a los controles (Figura 1). Es importante destacar que nuestro grupo de pacientes con tinnitus tuvo un promedio de edad 6 años mayor al de los controles (40 años versus 34 años). Si bien es conocido que los umbrales audiométricos de alta frecuencia se elevan con la edad ${ }^{32}$, la diferencia esperable para 6 años de diferencia va de 1 a $6 \mathrm{~dB}$ como máximo, por lo que las variaciones de cerca de $15 \mathrm{~dB}$ encontradas entre pacientes con tinnitus y controles no podrían ser explicadas por la diferencia de edad. Por otro lado, la elevación de los umbrales auditivos en altas frecuencias en sujetos con tinnitus y PTP normal ya había sido reportada por Vielsmeier et al. $(2015)^{33}$, por lo que nuestros datos confirman estos hallazgos. 


\section{Emisiones otoacústicas por producto de distorsión}

La amplitud de las EOAPD en el grupo con tinnitus fueron significativamente menores que las del grupo control en las frecuencias de 2786 y $3508 \mathrm{~Hz}$. Estos hallazgos concuerdan con trabajos anteriores, como Modh y cols. (2014) quienes reportaron EOAPD de menor amplitud en las frecuencias de 1560, 6250 y $8837 \mathrm{~Hz}^{34}$, mientras que otros autores como Shiomi y cols. (1997) describieron hallazgos similares en frecuencias entre 4 a $7 \mathrm{kHz}^{35}$. En nuestro caso, lamentablemente no estudiamos frecuencias de EOAPD mayores a $4 \mathrm{kHz}$, por lo que no podemos comparar en ese rango de frecuencias. Estos hallazgos evidencian la presencia de un daño coclear subclínico en este grupo de pacientes, lo que junto a la elevación de umbrales de alta frecuencia sugiere que en sujetos con tinnitus y PTP normal existe algún grado de desaferentación auditiva que probablemente originó el tinnitus ${ }^{36,37}$.

\section{Reflejo olivococlear con ruido contralateral}

No encontramos diferencias significativas entre pacientes con tinnitus y controles en la magnitud del efecto supresor del ROC sobre las EOAPD. Como ya se discutió en la introducción, estos hallazgos han sido heterogéneos en la literatura ${ }^{15,38}$, y en nuestro caso apoyaríamos al grupo de trabajo con resultados negativos. Una diferencia que podría explicar estas diferencias sería la hiperacusia. Knudson et al. (2014) encontraron que la hiperacusia es preponderante con respecto al tinnitus a la hora de explicar la variabilidad de los resultados del efecto del ROC en pacientes que presentan esta última afección ${ }^{39}$. Lamentablemente, una limitación de nuestro estudio es que no contamos con una medición sistemática de la hiperacusia. A futuro se debería medir en forma rutinaria la presencia de hiperacusia en pacientes con tinnitus y PTP normal. Además, se podría utilizar protocolos basados en emisiones otoacústicas por clics, en los que se ha visto un efecto supresor del ROC más confiable y repetible que con $\mathrm{EOAPD}^{40}$.

Es importante entender el circuito neuronal que explica los resultados obtenidos en nuestro estudio. Encontramos efectos significativos en el ROC medido en el oído izquierdo, pero evocado con ruido contralateral derecho.
La vía neuroanatómica de la estimulación del COSM con ruido contralateral incluye: cóclea derecha (contralateral), nervio auditivo derecho y núcleo coclear derecho, para cruzar la línea media y activar a neuronas olivococleares del COS izquierdo, que a su vez a través de los haces no cruzados inervan a las CCE del oído izquierdo para modular la amplitud de las EOAPD del oído izquierdo. La asimetría del ROC ya ha sido reportada previamente $e^{41,42}$ y podría relacionarse a la dominancia del oído derecho en la percepción auditiva. Así, se podría explicar el hecho de que encontramos efectos diferentes para el oído izquierdo comparado al derecho.

\section{Pruebas psicoemocionales (THI y STAI)}

El efecto supresor del ROC medido en el oído izquierdo fue significativamente menor en el grupo THI "moderado-severo" comparado al grupo "leve-muy leve". Estos resultados difieren de otros trabajos en que utilizaron una clasificación similar, como los desarrollados por Serra y cols. (2015) y Urnau y cols. (2012), sin embargo, estos trabajos utilizaron otro tipo de emisiones otoacústicas y no cuantificaron la magnitud del $\mathrm{ROC}^{16,43}$. Nuestros resultados sugieren que solo el grupo con un índice de THI de moderado a severo presentaría una disfunción del ROC del oído izquierdo, generando mayor hiperexcitabilidad de la vía auditiva y una decodificación errónea por parte de estructuras superiores del sistema auditivo central ${ }^{44}$. El test de ansiedad-rasgo no mostró diferencias significativas entre el grupo control y tinnitus. Este resultado fue sorpresivo, pero nos sugiere que los pacientes controles de nuestro estudio tuvieron también un elevado nivel de ansiedad, comparable al de los pacientes con tinnitus. Esto podría deberse al elevado nivel de estrés y ansiedad que tiene la mayoría de las personas en el estilo de vida moderno en las grandes ciudades.

No encontramos correlaciones significativas entre la amplitud de las EOAPD y las pruebas psicoemocionales (THI y STAI). Este resultado concuerda con lo reportado por Granjeiro y cols. (2013), quienes no lograron encontrar correlaciones entre la amplitud de EOAPD con los niveles de ansiedad y depresión en pacientes con tinnitus ${ }^{45}$. Por otro lado, encontramos una correlación positiva entre 
la magnitud del ROC del oído izquierdo y los puntajes del THI y del STAI para el grupo con molestias moderadas a severas. Estos datos mostraron que una mayor supresión a través del ROC del oído izquierdo se correlaciona con menos molestias psicoemocionales y menores niveles de ansiedad. La regulación de los niveles de ansiedad y peor calidad de vida generados por el tinnitus sobre la respuesta del efecto supresor del ROC se explicarían por conexiones noradrenérgicas del locus coeruleus con neuronas del COS, las que han sido descritas en modelos animales ${ }^{22,23}$. Las neuronas del LC interactuarían con las del COS durante la atención selectiva ${ }^{46}$, un proceso cognitivo que se encuentra afectado en pacientes con tinnitus ${ }^{47}$.

En base a los hallazgos del presente estudio y a los trabajos de Granjeiro y cols. $(2013)^{45}$, se podría concluir que el grado de severidad del tinnitus autopercibido por el paciente y la ansiedad no estarían asociados directamente con un deterioro de la función de las células ciliadas externas. Sin embargo, nuestras observaciones sugieren que el nivel de ansiedad en pacientes con tinnitus con un índice THI moderado a severo se correlaciona con la actividad del reflejo olivococlear medial que controla el amplificador coclear del oído izquierdo. Esto podría explicarse por la asimetría del procesamiento auditivo periférico, que se conoce como dominancia del oído derecho ${ }^{41,42}$. Sin embargo, debemos ser cautelosos con estos hallazgos, debido a la variabilidad de resultados observados entre diferentes publicaciones, ya sea por criterios en la selección de pacientes, metodología y análisis, entre otros factores. Por lo tanto, es importante la unificación y replicación de protocolos de estudios de este tipo, además aún es complejo precisar qué mecanismos específicos asocian la actividad del reflejo olivococlear con un aumento en los niveles de ansiedad.

Si bien, en este manuscrito no encontramos una relación significativa entre la magnitud del ROC-que corresponde a un reflejo del tronco encefálico-y tinnitus, esto no descarta la posibilidad de que las vías descendentes del sistema eferente auditivo que provienen de diferentes áreas de la corteza cerebral estén involucradas en la fisiopatología del tinnitus ${ }^{48,49}$. Por otro lado, la medición del ROC en pacientes con tinnitus podría tener un rol en aquellos con índices THI moderado, severo o catastrófico. Futuros trabajos que estudien la relación del sistema eferente auditivo y tinnitus debieran considerar la medición de ansiedad, hiperacusia y cuantificar la función de las vías eferentes auditivas que provienen de la corteza auditiva.

\section{Conclusión}

El aumento del nivel de ansiedad y autopercepción de empeoramiento de la calidad de vida en sujetos que sufren tinnitus en un grado moderado a severo está asociado a una disminución del efecto supresor del reflejo olivococlear medial medido en el oído izquierdo.

\section{Bibliografía}

1. Wimmer J, Donoso R, Leiva A, Breinbauer H, Délano P. Tinnitus: Una patología cerebral. Rev Otorrinolaringol Cir Cabeza Cuello. 2019;79:125-136.

2. Edmondson-Jones M, Mccormack A, Somerset S, Hall D. A systematic review of the reporting of tinnitus prevalence and severity. Hear Res. 2016; 337:70-79.

3. Kim HJ, Lee HJ, An SY, et al. Analysis of the prevalence and associated risk factors of tinnitus in adults. PLoS One. 2015;10:e0127578.

4. Langguth B. A review of tinnitus symptoms beyond 'ringing in the ears': a call to action. Curr Med Res Opin. 2011;27:1635-43.

5. Eggermont JJ, Tass PA. Maladaptive Neural Synchrony in Tinnitus: Origin and Restoration. Front Neurol. 2015;6:29.

6. Levine RA, Oron Y. Tinnitus. En: Celesia GG, Hickok G, EDS, Handbook of Clinical Neurology: The Human Auditory System. Fundamental Organization and Clinical Disorders. Edinburgh: Elsevier, 2015;129:409-31.

7. Singer W, Panford-Walsh R, Knipper M. The function of BDNF in the adult auditory system. Neuropharmacology. 2014; 76:719-728.

8. Concina G, Renna A, Grosso A, Sacchetti B: The auditory cortex and the emotional valence of sounds. Neurosci Biobehav Rev. 2019;98:256-264.

9. Terreros G, Délano PH. Corticofugal modulation of peripheral auditory responses. Front in Syst Neurosci. 2015; 9:134.

10. Mulders WH, Robertson D. Evidence for direct cortical innervation of medial olivocochlear neurons in rats. Hear Res. 2000;144:65-72.

11. Délano P, Robles I, Robles L. Sistema eferente 
auditivo. Rev Otorrinolaringol Cir Cabeza Cuello. 2005;65:55-62.

12. Guinan JJ. Olivocochlear Efferents: Anatomy, Physiology, Function, and the Measurement of Efferent Effects in Humans. Ear Hear. 2006;27:589607.

13. Aedo C, Tapia E, Pavez E, Elgueda D, Délano PH, Robles L. Stronger efferent suppression of cochlear neural potentials by contralateral acoustic stimulation in awake than in anesthetized chinchilla. Front Syst Neurosci. 2015;9:21.

14. Bowen M, Terreros G, Moreno-Gómez FN, et al. The olivocochlear reflex strength in awake chinchillas is relevant for behavioural performance during visual selective attention with auditory distractors. Sci Rep. 2020;10:14894. doi: 10.1038/s41598-020-71399-8.

15. Riga M, Katotomichelakis M, Danielides V. The Potential Role of the Medial Olivocochlear Bundle in the Generation of Tinnitus. Otol Neurotol. 2015;36:201-208.

16. Serra LS, Granjeiro RC, Braga SC, Oliveira CA, Sampaio AL. Association between suppression of otoacoustic emissions and annoyance levels in tinnitus patients with normal hearing. Int Tinnitus J. 2015;19:52-58.

17. Pattyn T, Van Den Eede F, Vanneste S, et al. Tinnitus and anxiety disorders: A review. Hear Res. 2016; 333:255-265.

18. Redmond DE Jr, Huang YH. Current concepts. II. New evidence for a locus coeruleus-norepinephrine connection with anxiety. Life Sci. 1979;25:2149-2162.

19. Pohl R, Rainey JM, Ortiz A, Yeragani VK. Locus coeruleus and anxiety. Biol Psychiatry. 1987;22:116117.

20. Tanaka M, Yoshida M, Emoto H, Ishii $H$. Noradrenaline systems in the hypothalamus, amygdala and locus coeruleus are involved in the provocation of anxiety: basic studies. Eur J Pharmacol. 2000;405:397-406.

21. Kaltenbach JA. The dorsal cochlear nucleus as a participant in the auditory, attentional and emotional components of tinnitus. Hear Res. 2006;216:224-234.

22. Mulders WHAM, Robertson D. Catecholaminergic innervation of guinea pig superior olivary complex. $J$ Chem Neuroanat. 2005a;30:230-242.

23. Mulders WHAM, Robertson D. Noradrenergic modulation of brainstem nuclei alters cochlear neural output. Hear Res. 2005b;204:147-155.

24. Salgado H, García-Oscos F, Dinh L, Atzori M. Dynamic modulation of short-term synaptic plasticity in the auditory cortex: The role of norepinephrine. Hear Res. 2011;271:26-36.

25. Pérez-Valenzuela C, Gárate-Pérez MF, Sotomayor-Zárate R, Délano PH, DagninoSubiabre A. Reboxetine Improves Auditory Attention and Increases Norepinephrine Levels in the Auditory Cortex of Chronically Stressed Rats.
Front Neural Circuits. 2016;10:108.

26. Newman GH, Jacobson GP, Spitzer JB. Development of the Tinnitus Handicap Inventory. Arch Otolaryngol Head Neck Surg. 1996;122:143-8.

27. Peña Martínez A. Evaluación de la incapacidad provocada por el tinnitus: homologación lingüística nacional del Tinnitus Handicap Inventory (THI). Rev Otorrinolaringol Cir Cabeza Cuello. 2006;66:232-235.

28. Der C, Alzérreca E, San Martín JT, et al. National linguistic validation of the Tinnitus Handicap Inventory (THI). Assessment of disability caused by tinnitus in Chilean Spanish-speaking population. Int Tinnitus J. 2012;17:146-151.

29. McCombe A, Baguley D, Coles R, et al. Guidelines for the grading of tinnitus severty: The results of a working group commisioned by the British Association of Otolaryngologists. Allied Sci. 2001;26:388-93.

30. Spielberger CD, Gorsuch RL, Lushene RE. STAI, Manual for the State-Trait Anxiety Inventory. California, Consulting Psychologists Press. Adapt. Española. Madrid, TEA Ediciones, 1982.

31. Vera-Villarroel P, Celis-Atenas K, Córdova-Rubio N, Buela-Casal G, Spielberger-Charles D. Preliminary Analysis and Normative Data of the State-Trait Anxiety Inventory (STAI) in Adolescent and Adults of Santiago, Chile. Ter Psicol. 2007; 25:155-162.

32. Lagos RG, López EM. Estudio normativo: Umbrales auditivos de alta frecuencia (9-20 $\mathrm{kHz}$ ) en normoyentes entre 8 años y 23 años y 11 meses, pertenecientes a la ciudad de Chillán. Rev Otorrinolaringol Cir Cabeza Cuello. 2016;76: 31-42.

33. Vielsmeier V, Lehner A, Strutz J, et al. The Relevance of the High Frequency Audiometry in Tinnitus Patients with Normal Hearing in Conventional PureTone Audiometry. Biomed Res Int. 2015;2015:302515.

34. Modh D, Katarkar A, Alam N, Jain A, Shah P. Relation of distortion product otoacoustic emission and tinnitus in normal hearing patients: A pilot study. Noise Health. 2014;16:69-72.

35. Shiomi Y, Tsuji J, Naito Y, Fujiki N, Yamamoto N. Characteristics of DPOAE audiogram in tinnitus patients. Hear Res. 1997;108:83-88.

36. Lucertini M, Moleti A, Sisto R. On the detection of early cochlear damage by otoacoustic emission analysis. J Acoust Soc Am. 2002;111:972-978.

37. Oeken J. Distortion Product Otoacoustic Emissions in acute acoustic trauma. Noise Health. 1998; 1:56-66.

38. Geven LI, de Kleine E, Free RH, van Dijk P. Contralateral suppression of otoacoustic emissions in tinnitus patients. Otol Neurotol. 2011;32:315-321.

39. Knudson IM, Shera CA, Melcher JR. Increased contralateral suppression of otoacoustic emissions indicates a hyperresponsive medial olivocochlear system in humans with tinnitus and hyperacusis. $J$ Neurophysiol. 2014;112:3197-3208.

40. Boothalingam S, Kurke J, Dhar S. Click-Evoked 
Auditory Efferent Activity: Rate and Level Effects. J Assoc Res Otolaryngol. 2018;19:421-434.

41. Khalfa S, Collet L. Functional asymmetry of medial olivocochlear system in humans. Towards a peripheral auditory lateralization. Neuroreport. 1996;7:993-996.

42. Khalfa S, Micheyl C, Veuillet E, Collet L. Peripheral auditory lateralization assessment using TEOAEs. Hear Res. 1998;121:29-34.

43. Urnau D, Tochetto, TM. Occurrence and suppression effect of Otoacoustic Emissions in normal hearing adults with tinnitus and hyperacusis. Braz J Otorhinolaryngol. 2012;78: 87-94.

44. Hinalaf M, Maggi AL, Hüg MX, Kogan P, Villalobo JP, Biassoni EC. Tinnitus, Medial Olivocochlear System, and Music Exposure in Adolescents. Noise Health. 2017;19:95-102.

45. Granjeiro RC, Kehrle HM, de Oliveira TS, Sampaio AL, de Oliveira CA. Is the degree of discomfort caused by tinnitus in normal-hearing individuals correlated with psychiatric disorders? Otolaryngol Head Neck Surg. 2013;148:658-663.

46. Yakunina N, Tae WS, Kim SS, Nam EC. Functional MRI evidence of the cortico-olivary efferent pathway during active auditory target processing in humans. Hear Res. 2019;379:1-11.

47. Heeren A, Maurage P, Perrot H, et al. Tinnitus specifically alters the top-down executive control sub-component of attention: evidence from the Attention Network Task. Behav Brain Res. 2014;269:147-154.

48. Elgueda D, Delano PH. Corticofugal modulation of audition. Curr Opinion Physiol 2020;18:73-78. doi:10.1016/j.cophys.2020.08.016.

49. Knipper M, van Dijk P, Schulze H, et al. The Neural Bases of Tinnitus: Lessons from Deafness and Cochlear Implants. J Neurosci. 2020;40:7190-7202. doi: 10.1523/JNEUROSCI.1314-19.2020. 